\title{
Universiteit
}

Leiden

The Netherlands

\section{Determination of curvature corrections to the surface tension of a liquid- vapor interface through molecular dynamics simulations}

Giessen, A.E. van; Blokhuis, E.M.

\section{Citation}

Giessen, A. E. van, \& Blokhuis, E. M. (2002). Determination of curvature corrections to the surface tension of a liquid-vapor interface through molecular dynamics simulations. Journal of Chemical Physics, 116(1), 302-310. doi:10.1063/1.1423617

Version: $\quad$ Publisher's Version

License: $\quad$ Leiden University Non-exclusive license

Downloaded from: https://hdl.handle.net/1887/79348

Note: To cite this publication please use the final published version (if applicable). 


\section{Determination of curvature corrections to the surface tension of a liquid-vapor interface through molecular dynamics simulations}

Cite as: J. Chem. Phys. 116, 302 (2002); https://doi.org/10.1063/1.1423617

Submitted: 08 May 2001. Accepted: 09 October 2001. Published Online: 17 December 2001

A. E. van Giessen, and E. M. Blokhuis

\section{ARTICLES YOU MAY BE INTERESTED IN}

Molecular dynamics study of the curvature correction to the surface tension

The Journal of Chemical Physics 100, 556 (1994); https://doi.org/10.1063/1.466972

The Statistical Mechanical Theory of Surface Tension

The Journal of Chemical Physics 17, 338 (1949); https://doi.org/10.1063/1.1747248

The Effect of Droplet Size on Surface Tension

The Journal of Chemical Physics 17, 333 (1949); https://doi.org/10.1063/1.1747247

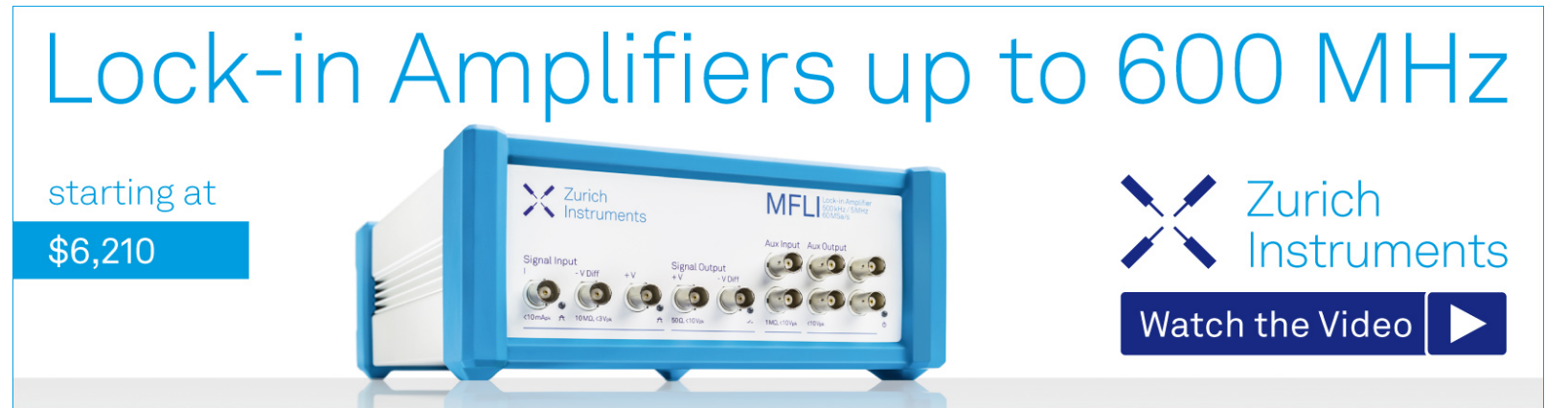




\title{
Determination of curvature corrections to the surface tension of a liquid-vapor interface through molecular dynamics simulations
}

\author{
A. E. van Giessen ${ }^{\mathrm{a})}$ and E. M. Blokhuis \\ Colloid and Interface Science, Leiden Institute of Chemistry, P.O. Box 9502, 2300 RA Leiden, \\ The Netherlands
}

(Received 8 May 2001; accepted 9 October 2001)

\begin{abstract}
We use molecular dynamics simulations of particles interacting through a truncated Lennard-Jones potential to study the surface properties of the curved liquid-vapor interface. We determine the Tolman length $\delta$, investigate its critical behavior, and provide first results for the rigidity constants of bending, $k$, and of Gaussian curvature, $\bar{k}$. The rigidity constant of bending, determined at three different temperatures, is found to be positive and of the order of one-half $k_{B} T$. The rigidity constant of Gaussian curvature, determined at a single temperature, is of the same order of magnitude.

(C) 2002 American Institute of Physics. [DOI: 10.1063/1.1423617]
\end{abstract}

\section{INTRODUCTION}

Complex interfaces are generally systems containing liquid surfaces in which at least one of the components has a strong affinity for the surface. Examples of complex interfaces include those occurring in systems containing surfactants (i.e., in microemulsions), lipids (i.e., membranes, vesicles), polymers (grafted or adsorbed to the surface), block-copolymers, etc. ${ }^{1}$ For the theoretical understanding of the properties of complex interfaces, it has long been recognized that the curvature properties play a dominant role. This recognition has led to the formulation of the surface curvature free energy by Helfrich which has the following form: ${ }^{2}$

$$
F=\int d A\left[\sigma-2 \frac{k}{R_{0}} J+\frac{k}{2} J^{2}+\bar{k} K\right] .
$$

The Helfrich free energy is derived by assuming that the radii of curvature, $R_{1}$ and $R_{2}$, are large compared to the length scales of interest so that an expansion in small curvature can be made. Specifically, the above free energy is an expansion to second order of the total curvature, $J=1 / R_{1}$ $+1 / R_{2}$ and Gaussian curvature, $K=1 /\left(R_{1} R_{2}\right)$, leading to the existence of four phenomenological coefficients: $\sigma$, the surface tension of the planar interface, $R_{0}$, the radius of spontaneous curvature, $k$, the rigidity constant associated with bending, and $\bar{k}$, the rigidity constant associated with Gaussian curvature.

The Helfrich expression for the surface free energy has been successfully applied to describe the shape and phase diagram of complex interfaces ${ }^{1,3}$ but also for the description of the properties of the simple liquid-vapor interface. ${ }^{4} \mathrm{~A}$ major drawback of the Helfrich description is that no information is provided on the value of the coefficients $\sigma, R_{0}, k$ and $\bar{k}$. Much theoretical attention has therefore focused on the determination of these phenomenological parameters in terms of microscopic models, e.g., membranes, vesicles, polymer systems, ${ }^{5}$ and microemulsion systems. ${ }^{3}$

a)Electronic mail: a.vgiessen@chem.leidenuniv.nl
Although our ultimate goal is to obtain a better understanding of complex interfaces, in this article we determine the curvature coefficients of a simple interface, i.e., the liquid-vapor interface of a one-component system, using molecular dynamics simulations. The reason to focus on simple interfaces is twofold. First, it turns out that the determination of the rigidity constants for the one-component system is already rather difficult. Second, the concept of the Helfrich free energy has not been free of controversy for simple interfaces. In his plenary lecture ${ }^{6}$ at the second Liquid Matter Conference in 1993, John Rowlinson asked the participants, "What could apparently be simpler than a drop of liquid?" Rowlinson then proceeded to argue that a number of key problems remain, the most persistent of which are those related to the existence of terms beyond $1 / R$-with $R$ the radius of the droplet - of the radius dependent surface tension $\sigma(R)$ : There remains the (..) more difficult question-do terms in higher powers than $1 / R$ in the expansion of $\Omega$ or $\sigma(R)$ have any meaning for a simple fluid? "There are strong arguments against their validity...." Rowlinson then proceeded to enumerate a number of arguments against continuing the expansion beyond $1 / R$. For a spherical liquid droplet and for a cylindrical liquid column, each with equimolar radius $R$, Eq. (1.1) reduces to

$$
\begin{aligned}
& \sigma_{s}(R) \equiv \frac{F_{s}}{A}=\sigma-4 \frac{k}{R_{0}} \frac{1}{R}+(2 k+\bar{k}) \frac{1}{R^{2}}+\cdots, \\
& \sigma_{c}(R)=\sigma-2 \frac{k}{R_{0}} \frac{1}{R}+\frac{k}{2} \frac{1}{R^{2}}+\cdots .
\end{aligned}
$$

Here, and throughout this article, the subscript " $s$ " indicates spherical geometry, while " $c$ " indicates cylindrical geometry; the dots represent terms of higher order than $1 / R^{2}$ in the expansion in the curvature. The first-order correction to the surface tension due to the curvature energy defines the socalled Tolman length, ${ }^{7} \delta$, which is related to the radius of spontaneous curvature via $\sigma \delta=2 k / R_{0}$. The doubts raised by Rowlinson concern the existence of the second-order 
terms - the rigidity constants $k$ and $\bar{k}$-for a simple liquid interface. In this article we address these doubts by calculating the rigidity constants using computer simulations.

It is important to note that the surface tension of the planar interface, which is measured in surface tension experiments, and the Tolman length are independent of the choice of the dividing surface. The rigidity constants $k$ and $\bar{k}$, however, do depend on this choice. This fact does not render the rigidity constants useless; it does, however, indicate the need to state which convention for locating the dividing surface is used. Two common choices are the equimolar surface, for which the excess number density is zero, and the "surface of tension," which makes the Laplace equation valid at all $R{ }^{8}$ The planar limit of the difference between these two dividing surfaces equals the Tolman length, $\delta=z_{e}-z_{s}$. There is no fundamental reason to choose one location of the dividing surface over the other-as long as they are chosen "sensibly coincident" " with the interfacial region. In this article we have chosen the equimolar surface as the dividing surface as a matter of convenience.

As a model system for our simulations, we use a onecomponent liquid-vapor system in which the particles interact through a truncated Lennard-Jones potential-at fixed volume, number of particles, and temperature. For this model system the phase diagram and some surface properties such as surface tension are well documented. ${ }^{10,11}$ Furthermore, we establish more accurately the Tolman length for the LennardJones system, since its sign, magnitude, and critical behavior have been the topic of much recent research. ${ }^{12}$

The derivation of expressions for the rigidity constants suitable for use in molecular dynamics simulations is given in Sec. II. Section III follows with detail of the simulations, while in Sec. IV we describe and discuss the results of those simulations. We end with a summary in Sec. V.

\section{VIRIAL EXPRESSIONS}

The way one usually determines the surface tension by computer simulations is through the "virial expression" first derived by Kirkwood and Buff in 1949. ${ }^{13}$ It expresses the surface tension in terms of the interaction potential $\phi(r)$ and the pair density of the planar interface $\rho_{0}^{(2)}\left(z_{1}, z_{2}, r\right)^{13}$

$$
\sigma=\frac{1}{4} \int d z_{1} \int d \mathbf{r}_{12} \phi^{\prime}(r) r\left(1-3 s^{2}\right) \rho_{0}^{(2)}\left(z_{1}, z_{2}, r\right),
$$

where $r \equiv\left|\mathbf{r}_{12}\right|, s \equiv \cos \theta_{12}$ and $z_{2} \equiv z_{1}+s r$. Similar expressions have been derived for the radius of spontaneous curvature (or Tolman length) and the rigidity constants. ${ }^{4}$ For the Tolman length, it was derived that ${ }^{4}$

$$
\begin{aligned}
\delta= & -\frac{1}{8 \sigma} \int d z_{1} \int d \mathbf{r}_{12} \phi^{\prime}(r) r\left(1-3 s^{2}\right) \\
& \times\left(z_{1}+z_{2}\right) \rho_{0}^{(2)}\left(z_{1}, z_{2}, r\right),
\end{aligned}
$$

where it is understood that the integration over $z$ runs from the liquid to the vapor phase and where the $z=0$ plane is chosen to coincide with the equimolar surface. ${ }^{8}$ For the cal- culation of the Tolman length, we also used an equivalent expression introduced by Haye and Bruin ${ }^{14}$

$$
\begin{aligned}
\delta= & \frac{1}{\sigma} \int d z z\left[p_{N}(z)-p_{T}(z)\right]-\frac{1}{12 \sigma} \int d z \int d \mathbf{r}_{12} \phi^{\prime}(r) r \\
& \times\left(1-3 s^{2}\right) z_{12} \rho_{0}^{(2)}\left(z_{1}, z_{2}, r\right) .
\end{aligned}
$$

The first term in this expression is known as the mechanical Tolman length, and involves the normal and transverse components to the pressure tensor, $p_{N}(z)$ and $p_{T}(z)$, respectively. by $^{4}$

The virial expression for the rigidity constants are given

$$
\begin{aligned}
k= & -\frac{1}{8} \int d z_{1} \int d \mathbf{r}_{12} \phi^{\prime}(r) r\left[\left(z_{1} z_{2}\left(1-3 s^{2}\right)\right.\right. \\
& \left.+\frac{r^{2}}{8}\left(1+6 s^{2}-15 s^{4}\right)\right) \rho_{0}^{(2)}\left(z_{1}, z_{2}, r\right) \\
& \left.+2\left(z_{1}+z_{2}\right)\left\{s^{2}-\sin ^{2} \varphi\left(1-s^{2}\right)\right\} \rho_{c, 1}^{(2)}\left(z_{1}, z_{2}, \varphi, r\right)\right] \\
\bar{k}= & \frac{1}{8} \int d z_{1} \int d \mathbf{r}_{12} \phi^{\prime}(r) r\left[\left(2 z_{1} z_{2}\left(1-3 s^{2}\right)\right.\right. \\
& \left.+\frac{r^{2}}{12}\left(3+6 s^{2}-25 s^{4}\right)\right) \rho_{0}^{(2)}\left(z_{1}, z_{2}, r\right) \\
& +\left(z_{1}+z_{2}\right)\left(\left(1-3 s^{2}\right) \rho_{s, 1}^{(2)}\left(z_{1}, z_{2}, r\right)\right. \\
& \left.\left.+4\left\{s^{2}-\sin ^{2} \varphi\left(1-s^{2}\right)\right\} \rho_{c, 1}^{(2)}\left(z_{1}, z_{2}, \varphi, r\right)\right)\right]
\end{aligned}
$$

where $\varphi \equiv \varphi_{12}$ is the angle between $\mathbf{r}_{1}$ and $\mathbf{r}_{2}$.

It has been noted ${ }^{4,6}$ that the expressions for the rigidity constants diverge when the potential decays as $r^{-6}$, or slower, at large $r$. This means that for a true Lennard-Jones fluid in which the potential is not truncated, the rigidity constants are infinite. In real systems, however, the potential crosses over to an $r^{-7}$ decay for large distances due to retardation effects, so that the above expressions for the rigidity constants lead to finite values. Also, in our simulations the rigidity constants are finite since the Lennard-Jones potential is truncated at $2.5 \sigma$. Even so, for real systems or, in general, for systems interacting through an algebraically decaying potential, the coefficients for higher order terms may very well diverge. At that point, the expansion is no longer analytic and terms containing, for example, $\ln (R)$ may need to be included. This does not, however, render the earlier terms meaningless since they are still dominant for large $R$.

The virial expressions for $\sigma$ and $\delta$ feature the pair density of the planar interface, $\rho_{0}^{(2)}\left(z_{1}, z_{2}, r\right)$, and can therefore be determined from a simulation of the planar interface only. The expressions for the rigidity constants, however, depend also on the leading order change in pair density of the spherical and cylindrical interface due to curvature

$$
\begin{aligned}
& \rho_{s}^{(2)}\left(z_{1}, z_{2}, r\right)=\rho_{0}^{(2)}\left(z_{1}, z_{2}, r\right)+\rho_{s, 1}^{(2)}\left(z_{1}, z_{2}, r\right) \frac{1}{R}+\cdots, \\
& \rho_{c}^{(2)}\left(z_{1}, z_{2}, \varphi, r\right)=\rho_{0}^{(2)}\left(z_{1}, z_{2}, r\right)+\rho_{c, 1}^{(2)}\left(z_{1}, z_{2}, \varphi, r\right) \frac{1}{R}+\cdots .
\end{aligned}
$$


For the determination of the rigidity constants, it is therefore essential to perform simulations of curved interfaces with very large radii of curvature so that the higher order terms in the expansion in $1 / R$ may be neglected. Furthermore, in order to derive expressions for each of the rigidity constants, it is necessary to consider two different geometries: the spherical and cylindrical interface.

We now discuss the way in which these virial expressions can be used in a molecular dynamics simulation to determine the various quantities. The surface tension and Tolman length are straightforwardly determined by evaluating the following expressions using simulations of a planar interface:

$$
\begin{aligned}
& \sigma=\frac{1}{4 A} \sum_{i, j} \phi^{\prime}\left(r_{i j}\right) r_{i j}\left(1-3 s^{2}\right), \\
& \delta=-\frac{1}{8 A \sigma} \sum_{i, j} \phi^{\prime}\left(r_{i j}\right) r_{i j}\left(1-3 s^{2}\right)\left(z_{i}+z_{j}\right),
\end{aligned}
$$

where $A$ is the surface area and the summation runs over all pairs of particles $i$ and $j$. The evaluation of the rigidity constants is somewhat more elaborate since it involves the leading order change in pair density with curvature. In the appendix we define the following integrals:

$$
\begin{aligned}
I_{s}(R) \equiv & \frac{1}{12 A} \int d \mathbf{r}_{1} \int d \mathbf{r}_{2} \phi^{\prime}(r) r \rho_{s}^{(2)}\left(\mathbf{r}_{1}, \mathbf{r}_{2}\right) \\
& \times\left\{-3\left(z_{1}+\frac{1}{2} s r\right)\left(1-3 s^{2}\right)\right. \\
& +\left[6 z_{1}^{2}\left(1-3 s^{2}\right)+z_{1} s r\left(15-27 s^{2}\right)\right. \\
& \left.\left.-\frac{r^{2}}{4}\left(3-42 s^{2}+55 s^{4}\right)\right] \frac{1}{R}\right\}
\end{aligned}
$$

for the spherical droplet, and

$$
\begin{aligned}
I_{c}(R) \equiv & \frac{1}{12 A} \int d \mathbf{r}_{1} \int d \mathbf{r}_{2} \phi^{\prime}(r) r \rho_{c}^{(2)}\left(\mathbf{r}_{1}, \mathbf{r}_{2}\right) \\
& \times\left\{\left(2 z_{1}+s r\right)\left[s^{2}-\sin ^{2} \varphi\left(1-s^{2}\right)\right]\right. \\
& -\left[3 z_{1}^{2}\left[s^{2}-\sin ^{2} \varphi\left(1-s^{2}\right)\right]\right. \\
& +z_{1} \operatorname{sr}\left[3 s^{2}-7 \sin ^{2} \varphi\left(1-s^{2}\right)\right] \\
& -r^{2}\left(s^{4}-4 s^{2} \sin ^{2} \varphi\left(1-s^{2}\right)\right. \\
& \left.\left.\left.+\frac{1}{3} \sin ^{4} \varphi\left(1-s^{2}\right)^{2}\right)\right] \frac{1}{R}\right\}
\end{aligned}
$$

for the cylinder. We also show that, to $\mathcal{O}(1 / R)$, these integrals are related to the following expansion with the curvature parameters as coefficients:

$$
\begin{aligned}
& I_{s}(R)=\frac{2 k}{R_{0}}-(2 k+\bar{k}) \frac{1}{R}+\cdots, \\
& I_{c}(R)=\frac{2 k}{R_{0}}-k \frac{1}{R}+\cdots .
\end{aligned}
$$

The way to determine the rigidity constants is to carry out simulations of both spherical droplets and cylindrical columns of certain (large) radius $R$. From the (negative) slope of a plot of $I_{c}(R)$ and $I_{s}(R)$ versus $1 / R$, one determines $k$ and $2 k+\bar{k}$, respectively. We thereby assume that the radii of curvature are large enough so that the remaining terms of the expansion in Eq.(2.10) can be neglected.

\section{SIMULATION DETAILS}

The simulations used to evaluate the surface tension, the Tolman length, and the rigidity constants consisted of molecular dynamics simulations of particles interacting through the Lennard-Jones potential

$$
\phi_{\mathrm{LJ}}(r)=4 \varepsilon\left[\left(\frac{\sigma}{r}\right)^{12}-\left(\frac{\sigma}{r}\right)^{6}\right],
$$

where $\varepsilon$ is the depth of the potential well, and $\sigma$ is the molecular diameter (not to be confused with the surface tension). The potential is cut off at a distance of $r_{c}=2.5 \sigma$. In addition, the potential is shifted such that it is zero at the cutoff. Thus, the actual potential used in the simulations is

$$
\phi(r)= \begin{cases}\phi_{\mathrm{LJ}}(r)-\phi_{\mathrm{LJ}}\left(r_{c}\right) & r<r_{c} \\ 0 & r<r_{c}\end{cases}
$$

As usual for MD simulations, all quantities are reduced by the appropriate factors of $\varepsilon, \sigma$, Boltzmann's constant $k_{B}$, and the particle mass $m$. In general, due to the finite cutoff, the physical quantities obtained in the simulations are only approximations of those in real systems. It is known that the value of the surface tension changes substantially when the cutoff is increased from $2.5 \sigma$ to $7.33 \sigma .^{11,15}$ This effect is even more dramatic for the rigidity constants since the values of the rigidity constants diverge logarithmically when the cutoff grows to infinity. In real systems, the rigidity constants are finite due to retardation effects, but still one may wonder whether the values of the rigidity constants obtained here using a truncated Lennard-Jones potential with $r_{c}=2.5 \sigma$ are a good approximation of the value for realistic systems. It would certainly be worthwhile to investigate the dependence of the rigidity constants on the potential cutoff in more detail; to do so, however, is computationally very expensive and we leave this as a project for future work.

The initial configurations for the simulations contained both phases, a high-density slab, sphere, or cylinder surrounded by a low-density "vapor," with the particles on an fcc lattice. The lattice spacing was appropriate for a liquid or for a vapor density, depending on the phase. The desired geometry of the equilibrated phase was already present in the initial configuration. For the simulations with droplets or cyl- 
TABLE I. Parameters for spheres at $T^{*}=0.90$.

\begin{tabular}{crr}
\hline \hline Box size & $N$ & $R_{e}$ \\
\hline $120 \times 120 \times 120$ & 158999 & 30.7 \\
$100 \times 100 \times 100$ & 90600 & 25.3 \\
$80 \times 80 \times 80$ & 48884 & 20.9 \\
$60 \times 60 \times 60$ & 23140 & 16.8 \\
$50 \times 50 \times 50$ & 13108 & 13.7 \\
$50 \times 50 \times 50$ & 10048 & 11.0 \\
$50 \times 50 \times 50$ & 8750 & 9.4 \\
$40 \times 40 \times 40$ & 5195 & 8.7 \\
\hline \hline
\end{tabular}

inders, the volume of the high-density phase in the initial configuration was adjusted to achieve a predetermined number of particles, which then, when equilibrated, formed a sphere or cylinder of the desired radius. For systems with larger radii, the initial configurations were based on the equilibrated configuration of a system with a smaller radius, which was then padded with a spherical or cylindrical region of particles on an fcc lattice with a liquid-like density, and then the remainder of the volume was filled with a lattice of vapor-like density, until the target number of particles had been reached. All configurations were allowed to fully equilibrate, a process which could last for millions of time steps for large systems. The equilibration process for the large systems was sped up by rescaling the momenta in the dense phase separately from those in the vapor phase, which greatly reduced the equilibration time.

The paucity of simulations of cylinder in the literature ${ }^{16,17}$ initially led us to believe that such simulations were difficult. However, this turns out not to be the case; simulating cylindrical liquid columns is actually straightforward. It is simply a matter of a judicious choice of box shape and size: the dimension of the box parallel to the cylinder axis must be less than the diameter of the cylinder, while the two dimensions perpendicular to the axis should be much larger than the diameter. For the simulations reported here, the lengths of the box perpendicular to the cylinder axis were always at least four times the cylinder radius. One must also start the simulation with a cylindrical initial configuration. This configuration is extremely stable; only when the dimension parallel to the axis is considerably larger than the diameter does the cylinder break up into droplets. Only one cylinder evaporated in the simulations, that of the smallest system, $N=1714$, at the highest temperature, $T^{*}=0.95$; all other cylinders were stable.

The expressions for $I(R)$, Eqs. (2.8) and (2.9), contain an explicit reference to the equimolar radius, $R=R_{e}$, of the droplet or cylinder. In order to evaluate these integrals, we used five "test" radii, one of which, $R_{\text {test }}$, was the approximate equimolar surface determined from the equilibration run. The other four radii were $R_{\text {test }} \pm 0.1$ and 0.2. After the production run was finished, we determined the average equimolar radius and then interpolated to the correct value of $I\left(R_{e}\right)$ using a quadratic fit to the five values calculated for $R_{\text {test }}$ and $R_{\text {test }} \pm 0.1$ and 0.2 .

The simulations used both the link-list and the pair-list method. The lists were updated every seven time steps. The reduced time step was $\Delta t=0.01$. The temperature was kept
TABLE II. Parameters for cylinders at $T^{*}=0.90$.

\begin{tabular}{ccc}
\hline \hline Box size & $N$ & $R_{e}$ \\
\hline $120 \times 120 \times 12$ & 30006 & 30.6 \\
$120 \times 120 \times 12$ & 20000 & 22.4 \\
$80 \times 80 \times 12$ & 12250 & 19.2 \\
$80 \times 80 \times 12$ & 10000 & 16.4 \\
$80 \times 80 \times 12$ & 8700 & 14.6 \\
$60 \times 60 \times 12$ & 6050 & 13.0 \\
$60 \times 60 \times 12$ & 4177 & 9.4 \\
$40 \times 40 \times 12$ & 2227 & 7.4 \\
$40 \times 40 \times 12$ & 1714 & 5.6 \\
\hline \hline
\end{tabular}

constant by scaling the particle velocities every 100 time steps. The total momentum of the system was set to zero periodically, to correct for drift due to round-off errors in the calculation; this is more important for smaller systems than for larger systems. For small systems, the drift was removed every 100 time steps, while for larger systems, this was changed to every 200 or 400 time steps. The center of mass of the system was moved to the center of the box when the total momentum was set to zero to prevent drift in the location of the sphere or cylinder. The statistical error in various quantities was determined by averaging over subintervals of 100000 time steps. Typical runs were anywhere from 2 $\times 10^{6}$ to $4 \times 10^{6}$ time steps in length, though all runs of planar interfaces consisted of at least $4 \times 10^{6}$ time steps, increasing up to $1 \times 10^{7}$ for the two temperatures nearest the critical point. Simulations for the planar interface were run at nine different temperatures: $T^{*}=0.75,0.80,0.85,0.90,0.95$, $0.975,1.00,1.025$, and 1.05 ; those of cylinders were performed at temperatures of $T^{*}=0.80,0.90$, and 0.95 , while simulations of droplets were done at only one temperature, $T^{*}=0.90$. All simulations for the planar interfaces were run using a system containing either 7100 particles in a box of $20 \times 20 \times 80$, or, for $T^{*} \geqslant 0.975,11000$ particles in a box of $20 \times 20 \times 100$. Details of the systems containing droplets and cylinders can be found in Tables I and II, respectively.

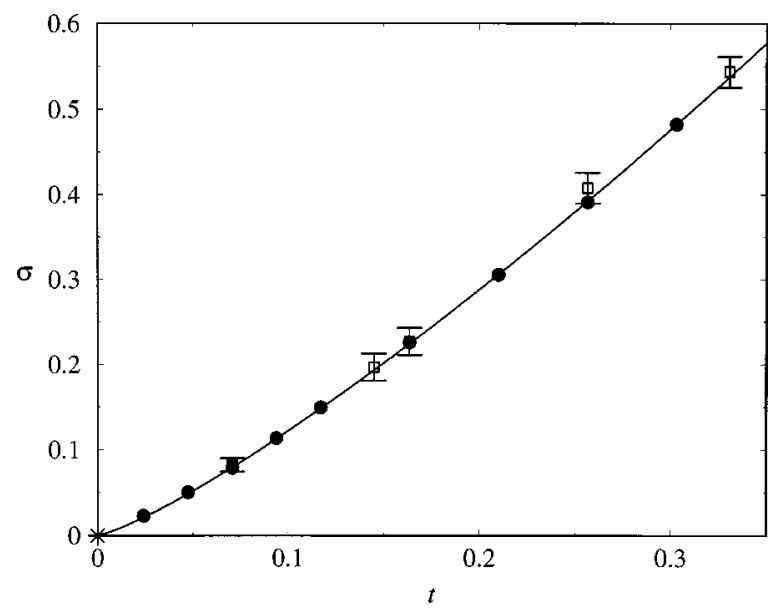

FIG. 1. A plot of the reduced surface tension $\sigma$ vs the reduced temperature $t \equiv\left(T_{c}-T\right) / T_{c}$; the error in each data point is smaller than the symbol. The solid line is a fit to our data (circles), and has an exponent of $\mu=1.24$. The critical temperature is indicated by the star. The squares are data from Ref. 10 , and are shown for comparison. 
TABLE III. Values for the number of time steps, the equilibrium densities, the surface tension, and the Tolman length at different temperatures.

\begin{tabular}{cccccc}
\hline \hline$T^{*}$ & Time steps & $\rho_{v}$ & $\rho_{\ell}$ & $\sigma_{0}$ & $\delta$ \\
\hline 1.050 & $1.18 \times 10^{7}$ & 0.158 & 0.490 & $0.023 \pm 0.002$ & $1.314 \pm 0.230$ \\
1.025 & $1.05 \times 10^{7}$ & 0.124 & 0.537 & $0.050 \pm 0.002$ & $0.931 \pm 0.157$ \\
1.000 & $8.0 \times 10^{6}$ & 0.101 & 0.570 & $0.079 \pm 0.002$ & $0.374 \pm 0.101$ \\
0.975 & $6.0 \times 10^{6}$ & 0.081 & 0.597 & $0.113 \pm 0.002$ & $0.332 \pm 0.081$ \\
0.95 & $4.0 \times 10^{6}$ & 0.067 & 0.621 & $0.150 \pm 0.002$ & $0.335 \pm 0.024$ \\
0.90 & $4.0 \times 10^{6}$ & 0.045 & 0.662 & $0.226 \pm 0.002$ & $0.264 \pm 0.030$ \\
0.85 & $4.0 \times 10^{6}$ & 0.031 & 0.697 & $0.306 \pm 0.002$ & $0.161 \pm 0.020$ \\
0.80 & $4.0 \times 10^{6}$ & 0.020 & 0.729 & $0.391 \pm 0.002$ & $0.169 \pm 0.018$ \\
0.75 & $4.0 \times 10^{6}$ & 0.013 & 0.758 & $0.483 \pm 0.002$ & $0.163 \pm 0.016$ \\
\hline \hline
\end{tabular}

\section{RESULTS AND DISCUSSION}

We begin by discussing the results from the planar interfaces. The equilibrium properties of the systems are listed in Table III. From the temperature-density coexistence curve, we find a critical temperature of $T_{c}^{*}=1.076$. Values for the calculated surface tension agree well with those found in the literature. ${ }^{10,11}$ Figure 1 shows our values plotted against the reduced temperature distance to the critical point, $t \equiv\left(T_{c}\right.$ $-T) / T_{c}$. For comparison, values from Ref. 10 are included. The critical temperature is also indicated by the star. A fit to the scaling law $\sigma \sim|t|^{\mu}$, shown as the solid line, gives a value for the critical scaling exponent of $\mu=1.24 \pm 0.02$. The accepted value of $\mu$ is $1.26{ }^{8}$

The Tolman length was calculated using Eqs. (2.2) and (2.3): the results from both expressions are in agreement, and are consistent with those of Haye and Bruin. ${ }^{14}$ The data, determined by fitting the values of $\delta$ calculated from each subinterval to a Gaussian distribution, are shown in Fig. 2 as a function of the reduced temperature distance to the critical point, $t$; they are also given in Table III. The Tolman length is always positive, and far from the critical point, it is approximately $0.16 \sigma$. Near the critical point, the Tolman length appears to diverge. The data from the two highest temperatures appear to be slightly inconsistent with the behavior shown at lower temperatures. Given the scatter in the data, we are not

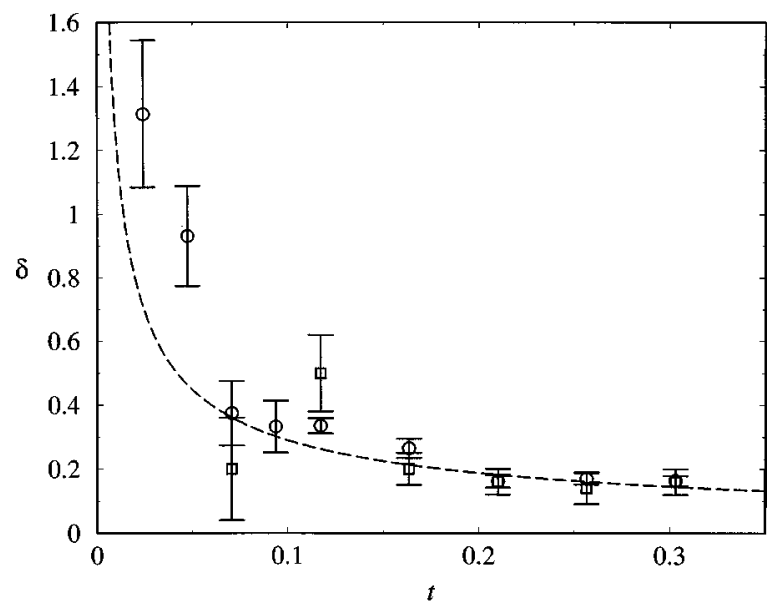

FIG. 2. The Tolman length, shown as circles, is plotted against the reduced temperature distance to the critical point, $t \equiv\left(T_{c}-T\right) / T_{c}$. The open squares are the data of Haye and Bruin (Ref. 14). The dashed line is a guide to the eye with an exponent of -0.63 . able to determine the critical exponent for the Tolman length. Physical arguments suggest that the magnitude of the critical exponent cannot be larger than $\nu=0.63$. This is because the Tolman length is the difference between two dividing surfaces, the equimolar dividing surface and the surface of tension, and since each of these surfaces lies within the interface, it would be expected that the Tolman length cannot diverge any faster than the width of the interface. The data from the lower temperatures (excluding the two highest temperatures) are not inconsistent with a critical exponent of -0.63 ; the dashed line in Fig. 2 is proportional to $t^{-0.63}$ and is a guide to the eye. The analyses of both Rowlinson ${ }^{18}$ and of Fisher and Wortis ${ }^{19}$ indicate that the divergence of the Tolman length would be dominated by either of two terms. One, due to field-mixing terms in the curvature correction in the renormalization group analysis, diverges as $|t|^{-0.06}$ as the critical temperature is approached. The other, which arises from the asymmetry of the local free-energy density, diverges as $|t|^{\theta_{5}-\nu}$. Fisher and Wortis were unable to determine $\theta_{5}$ due to poor convergence of the $\varepsilon$ expansion, though their analysis suggests it is positive. Equating -0.63 to $\theta_{5}$ $-\nu$ gives a value for $\theta_{5} \approx 0$.

Typical equilibrium configurations of a liquid droplet and a liquid column, both surrounded by their vapor, are shown in Figs. 3 and 4, respectively. The droplet has an equimolar radius of $8.7 \sigma$, while the cylinder has an equimo-

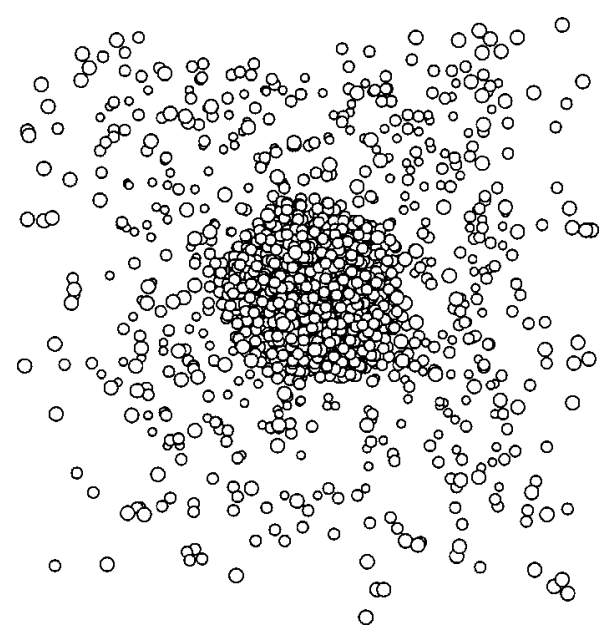

FIG. 3. A typical configuration for a spherical liquid droplet surrounded by its vapor. The radius of the droplet is $8.7 \sigma$. 


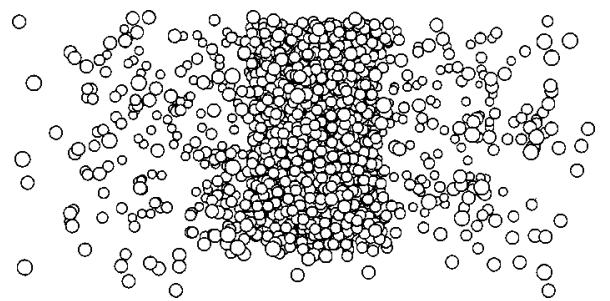

FIG. 4. A typical configuration for a cylindrical liquid column in its vapor. The radius of the cylinder is $6.2 \sigma$.

lar radius of $6.2 \sigma$. The system sizes considered in these simulations ranged up to $N=30000$ for columns, and up to 160000 for droplets. Tables I and II contain the number of particles, the equimolar radius, and the box dimensions for the simulations at $T^{*}=0.9$. Simulations at other temperatures used the same number of particles and box size, while the radius was allowed to vary. Larger systems were considered, for example a cylindrical column of $N=61000$ with a radius $R_{e} \approx 50 \sigma$, but the calculation of $I_{c}(R)$ proved to be unreliable. This is due to the presence of large capillary wave fluctuations along the surface. Figure 5 shows a projection of a typical configuration of this large cylinder onto the $x-y$ axis, with the center of each particle represented as a dot. One can clearly see the fluctuations in the location of the surface. These fluctuations reduce the radial symmetry of the system, which is reflected in an increase of the (average) interfacial width and a decrease in the accuracy of the calculation of $I(R)$, which depends explicitly on a known and constant $R_{e}$. This provides a practical upper limit for the size of the system. We do not include any calculations with a radius greater than $R=32$.

In order to investigate any finite-size effects due to cutting off the length of the cylinder in the $z$ direction, we ran two pairs of simulations of cylinders of identical radii, but with different box lengths in the $z$ direction. For each pair, the smaller system had a $z$ dimension of $10 \sigma$, while the larger system had one of $20 \sigma$. The larger system was exactly twice the size of the smaller system: twice the number of

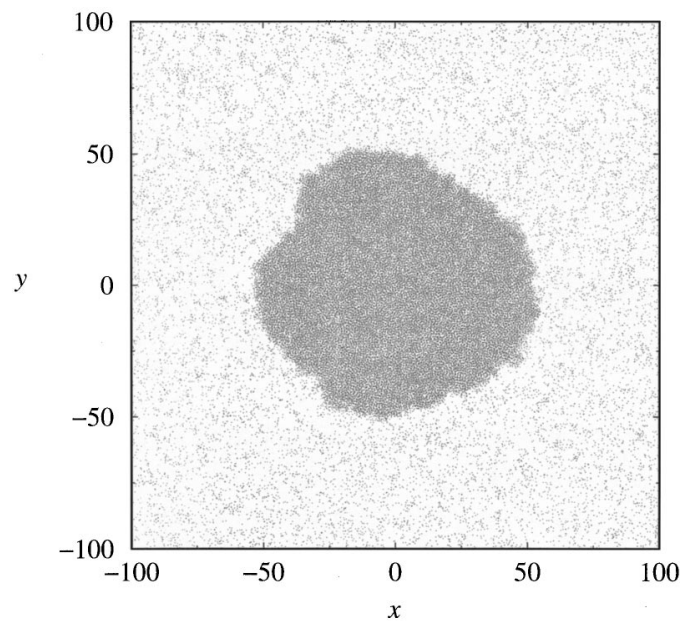

FIG. 5. A projection onto the $x-y$ axis of a cylinder with a radius of 50. The center of each particle is represented by a dot. Note the clearly visible capillary waves.

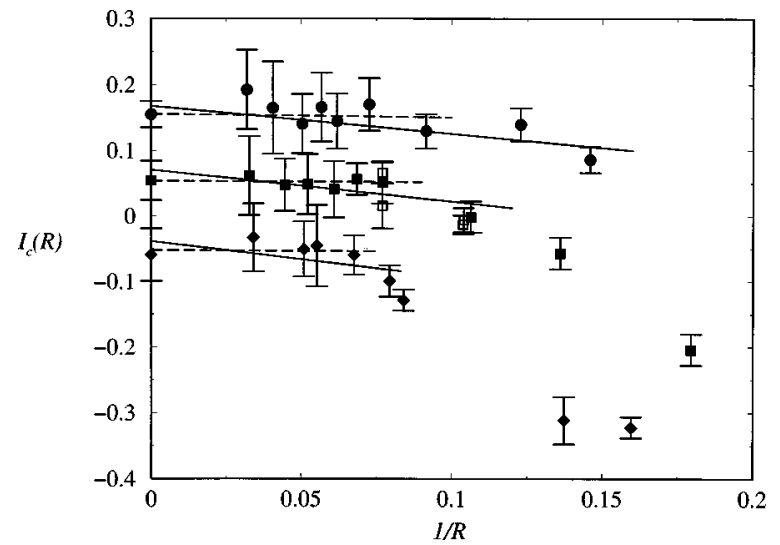

FIG. 6. Values for $I_{c}(R)$ are plotted vs $1 / R$ for three temperatures: $T^{*}$ $=0.80$ (circles), 0.90 (squares), and 0.95 (diamonds). For clarity, the data for $T^{*}=0.80$ have been shifted up by 0.1 , while those for $T^{*}=0.95$ have been shifted down by 0.1 . The various linear fits are described in the text. The open squares are results with different box lengths in the $z$ direction; the lower of the each pair has a length of $10 \sigma$.

particles and twice the volume. One pair had an equimolar radius of $R_{e}=12.99 \pm 0.03$; the larger system had 12772 particles and the smaller system had 6386 particles. The other pair had $R_{e}=9.62 \pm 0.03$, with the larger system containing 7200 particles, and the smaller, 3600 particles. For both pairs, the bulk densities are in agreement. The values for $I_{c}(R)$ differ slightly, with the smaller system having a smaller $I_{c}(R)$, though they agree to within the margin of error. In comparison with the data presented below, which all have a box length in the $z$ direction of $12 \sigma$, there is no obvious dependence of $I_{c}(R)$ on the box length, other than the data for systems with a box length in the $z$ direction of $10 \sigma$ being the lowest. The data from the systems discussed here are shown in Fig. 6 as open squares.

To determine the coefficients in the expansions (A6) and $(\mathrm{A} 7)$, we plot $I_{c}(R)$ versus $1 / R$ in Fig. 6 and $I_{s}(R)$ versus $1 / R$ in Fig. 7. Figure 6 contains data for three temperatures: $T^{*}=0.80$ (circles), 0.90 (squares), and 0.95 (diamonds). The data for $T^{*}=0.80$ and $T^{*}=0.95$ have been shifted vertically for clarity. The intercept, $2 k / R_{0}$, is also equal to $\sigma \delta$, where $\delta$ is the Tolman length; both $\sigma$ and $\delta$ were determined from simulations of planar interfaces, as described above. In Fig. 6 , one can see that for large $R$ the data for all three temperatures are independent of $R$, to within the margin of error. This regime includes data for systems with radii of $R=12 \sigma$ $(1 / R=0.083)$ and larger. For smaller values of the radius, the value for $I_{c}(R)$ decreases with decreasing radii. The rate of this decrease depends on the temperature: For the lowest temperature, $I_{c}(R)$ decreases only slightly as $R$ decreases, while for the highest temperature, the decrease in $I_{c}(R)$ is very rapid with decreasing $R$.

To extract the rigidity constant $k$ from these data, it is necessary to fit Eq. (2.9) to the "large- $R$ " regime. However, just where this regime ends is unclear. Applying linear regression to the first five (for $T^{*}=0.95$ ) or seven (for $T^{*}$ $=0.90$ and 0.80 ) data points gives values for $k$ which are indistinguishable from zero, to within the margin of error. This is the region where the data in Fig. 6 are obviously linear, i.e., for systems with radii of $12 \sigma$ and larger. These 


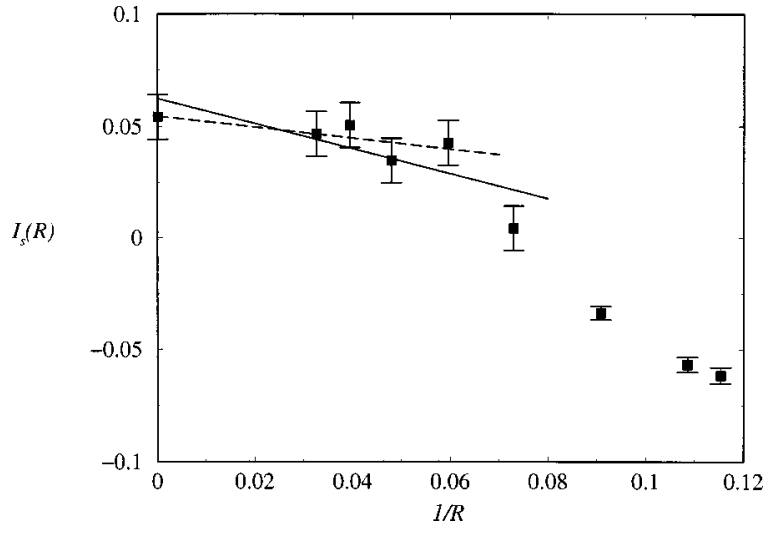

FIG. 7. $I_{s}(R)$ vs $1 / R$ for $T^{*}=0.90$. The two fits described in the text are shown as solid lines.

fits are shown as dashed lines. From a physical standpoint, however, cylinders with radii of $10 \sigma$ are already quite large and would be expected to be within the large- $R$ limit. Consequently, we can extend the large- $R$ regime to include data that lie just outside the linear region but nevertheless represent large cylinders. This means including data in the region where $I_{c}(R)$ just begins to curve towards more negative values. Fitting Eq. (2.9) to this extended region, shown as solid lines in Fig. 6, results in nonzero values for the rigidity constant. Note that these lines fall within the error for all data considered. These values for $k$ are all on the order of one half; the exact values are given in Table IV. We also see a slight increase of $k$ with temperature. Given the difficulty of the fit, these values can only be seen as estimates for $k$. In any case, it seems safe to say that the rigidity constant is positive, and less than 1.0 for all temperatures.

Figure 7 shows the data for $I_{s}(R)$ for various spherical droplets at a single temperature, $T^{*}=0.90$. The linear regime extends to approximately $R=16(1 / R=0.063)$, which is larger than that of the cylinder at the same temperature, approximately $R=12$. This is consistent with the expectation that the linear regime for the spheres should begin at a larger $R$ than for the cylinders, since for a given $R$, the curvature of a sphere is greater than that of a cylinder. A strategy similar to that used above for determining values for $k$ results in two values for $2 k+\bar{k}$, corresponding to the dashed and solid lines in Fig. 7. As an estimate for $\bar{k}$, we take the average of the two fits giving a value of $2 k+\bar{k}=0.40 \pm 0.20$. Using this and the values for $k$ determined above, gives $\bar{k}=-0.57 \pm 0.31$ using the extended region $(\bar{k} \approx 0.40 \pm 0.52$ for the strictly linear region).

In order to facilitate the comparison of our results with those from experiments and from other calculations, we can use the value of $\varepsilon / k_{B} T=119.4$ for $\operatorname{argon}^{8}$ to estimate $k$ and $\bar{k}$ in terms of $k_{B} T$; this results in an estimate $k \approx 0.5 k_{B} T$ for all temperatures, though again increasing slightly with temperature, and an estimate of the magnitude of $\bar{k} \approx-0.5 k_{B} T$. The calculated value of $k$ is positive, which is in contrast to mean-field calculations, ${ }^{4,20}$ which indicate that $k$ is negative. In comparison with experimental results, both Meunier et $a .^{21,22}$ and $\mathrm{Schmidt}^{23}$ have determined $k$ to be positive and
TABLE IV. Fit parameters and values for $k$ and $\bar{k}$.

\begin{tabular}{crcc}
\hline \hline$T^{*}$ & $N_{\text {fit }}$ & $k$ & $\bar{k}$ \\
\hline 0.95 & 6 & $0.55 \pm 0.20$ & \\
0.90 & 8 & $0.49 \pm 0.15$ & $-0.57 \pm 0.31$ \\
0.80 & 10 & $0.42 \pm 0.10$ & \\
\hline \hline
\end{tabular}

approximately $1 k_{B} T$ for an AOT monolayer and for a binary liquid-liquid mixture, respectively. In recent x-ray scattering experiments by Daillant and co-workers on water and organic liquids, ${ }^{24}$ it was shown that the surface energy is lowered at small wavelengths, which indicates a negative rigidity constant. These results were shown to be consistent with calculations by Mecke and Dietrich. ${ }^{25}$ Although it thus seems that our MD results are in contradiction with mean-field calculations ${ }^{4}$ and the scattering experiments, ${ }^{24}$ it might very well be that the rigidity constant describing fluctuations of a planar interface differs from the rigidity constant describing the equilibrium shape of curved surfaces. ${ }^{20}$.

\section{SUMMARY}

We have performed molecular dynamics simulations of a simple Lennard-Jones fluid, and have calculated surface properties for both planar and curved interfaces. The calculated surface tension of the planar interface agrees well with values found in the literature. ${ }^{10} \mathrm{We}$ also calculate the Tolman length, and find that it is positive and approximately $\delta$ $\approx 0.16 \sigma$ far from the critical point, and increases as the critical temperature is approached. We were unable to determine the precise critical behavior of the Tolman length, but our results are not inconsistent with a critical exponent of $-\nu$ $=-0.63$.

We also present the first calculations of the rigidity constants $k$ and $\bar{k}$ from simulations of spherical and cylindrical interfaces. These calculations involve simulating both spherical droplets and cylindrical columns for various large radii. Our results are summarized in Table IV. We find $k$ to be positive and on the order of one-half $k_{B} T$; we also find $k$ increases slightly with increasing temperature. The magnitude of $k$ is a factor of 2 (or more) smaller than that determined from experiments. ${ }^{21,23}$ Due to the difficult nature of the calculation, we are less certain of the sign of $\bar{k}$ and estimate its magnitude at $|\bar{k}|<k_{B} T$.

The existence of the rigidity constants for a simple fluid such as our Lennard-Jones fluid is not without controvery. Rowlinson ${ }^{6}$ has summarized many of the concerns about the validity of the expansion of the free energy past the order of $1 / R$. As mentioned above, his concern that the expressions for the rigidity constants diverge when the intermolecular potential decays as $r^{-6}$ for large $r$ does not apply, since the potential used in these simulations has a definite short-range cutoff. For the same reason, we do not expect to see, nor do we see, the expansion breakdown due to nonanalytic terms such as $\ln (R)$. It is difficult to address Rowlinson's remaining concerns, since they are of a more general nature and cannot be investigated via molecular dynamics simulations. The main question, however, is that of the physical relevance of 
the expansion coefficients when applied to interfaces of a simple fluid. We see no a priori reason to doubt their relevance. We would expect them to be small in magnitude, which we indeed find from our simulations, since the particles in our simulations have no internal structure. Even were they to be zero, a conclusion which could be supported with our results, they would still offer insight into the physical behavior of the surface: that an interface of a simple fluid has little or no rigidity. The calculation of these coefficients does indeed depend on the choice of the dividing surface, but this does not diminish their usefulness.

\section{ACKNOWLEDGMENTS}

M. Haye and C. Bruin are gratefully acknowledged for providing us with their original molecular dynamics code. The authors acknowledge financial support from N. W. O. Chemische Wetenschappen.

\section{APPENDIX: THE FUNCTIONS $I_{s}(R)$ AND $I_{c}(R)$}

In this appendix we show how the integrals as defined in Eqs. (2.8) and (2.9) are related to the curvature expansion in Eq. (2.10). This is done by reinvestigating the derivation of the virial expressions for the rigidity constants in Ref. 4. These virial expression are derived by evaluating the change in free energy under two deformations of the interface, each of which preserves the volume of the phases, but changes the radius $R$ by an infinitesimal amount $\delta R$. Full details of these transformations are given in Ref. 4. On the one hand, the general formula for the change in free energy, $\delta F$, is given by the statistical mechanical expression

$$
\delta F=\frac{1}{2} \int d \mathbf{r}_{1} \int d \mathbf{r}_{2}\left(\delta \mathbf{r}_{2}-\delta \mathbf{r}_{1}\right) \cdot \mathbf{r}_{12} \frac{\phi^{\prime}(r)}{r} \rho^{(2)}\left(\mathbf{r}_{1}, \mathbf{r}_{2}\right),
$$

while on the other hand it can be written in terms of the surface tension $\sigma(R)$ and its derivative $C(R) \equiv(\partial \sigma / \partial R)$. We now discuss these two approaches in more detail.

It is shown in Ref. 4 that for the specific transformation considered, labeled 1 and 2, the change in free energy for the spherical interface is given by

$$
\begin{aligned}
& \delta F_{1}=-A\left[\frac{\sigma_{s}(R)}{R}-C_{s}(R)\right] \delta R, \\
& \delta F_{2}=2 A\left[\frac{\sigma_{s}(R)}{R}+\frac{1}{2} C_{s}(R)\right] \delta R,
\end{aligned}
$$

and for the cylinder by

$$
\begin{aligned}
& \delta F_{1}=-A\left[\frac{\sigma_{c}(R)}{R}-C_{c}(R)\right] \delta R, \\
& \delta F_{2}=A\left[\frac{\sigma_{c}(R)}{R}+C_{c}(R)\right] \delta R .
\end{aligned}
$$

By taking the appropriate combinations of Eqs. (A2) and (A3), and of Eqs. (A4) and (A5), we can eliminate $\sigma(R)$ and obtain

$$
\begin{aligned}
\frac{R^{2}}{6 A \delta R}\left(2 \delta F_{1}+\delta F_{2}\right) & =\frac{R^{2}}{2} C_{s}(R) \\
& =\frac{2 k}{R_{0}}-(2 k+\bar{k}) \frac{1}{R}+\cdots
\end{aligned}
$$

for the spherical interface, and

$$
\frac{R^{2}}{2 A \delta R}\left(\delta F_{1}+\delta F_{2}\right)=R^{2} C_{c}(R)=\frac{2 k}{R_{0}}-k \frac{1}{R}+\cdots
$$

for the cylindrical interface. The left-hand side of these expansion can also be evaluated using the statistical mechanical expression in Eq. (A1). Beginning with the sphere, we have, for the first transformation ${ }^{20}$

$$
\begin{aligned}
\left(\delta \mathbf{r}_{2}-\delta \mathbf{r}_{1}\right) \cdot \mathbf{r}_{12}= & -\frac{1}{2} \frac{\delta R}{R} r^{2}\left[\left(1-3 s^{2}\right)-\frac{3 s r}{r_{1}}\left(1-s^{2}\right)\right. \\
& \left.-\frac{3 r^{2}}{4 r_{1}^{2}}\left(1-s^{2}\right)\left(1-5 s^{2}\right)\right],
\end{aligned}
$$

and for the second transformation ${ }^{20}$

$$
\begin{aligned}
\left(\delta \mathbf{r}_{2}-\delta \mathbf{r}_{1}\right) \cdot \mathbf{r}_{12}= & \frac{\delta R}{R} r^{2}\left(\frac{R}{r_{1}}\right)^{3}\left[\left(1-3 s^{2}\right)-\frac{3 s r}{2 r_{1}}\left(3-5 s^{2}\right)\right. \\
& \left.-\frac{r^{2}}{2 r_{1}^{2}}\left(3-30 s^{2}+35 s^{4}\right)\right] .
\end{aligned}
$$

For the cylinder, these are, using the notation in Ref. 4

$$
\begin{aligned}
\left(\delta \mathbf{r}_{2}-\delta \mathbf{r}_{1}\right) \cdot \mathbf{r}_{12}= & \frac{\delta R}{R} r^{2}\left[s^{2}-\sin ^{2} \varphi\left(1-s^{2}\right)\right. \\
& +\frac{2 s r}{r_{1, \|}} \sin ^{2} \varphi\left(1-s^{2}\right)-\frac{2 r^{2}}{3 r_{1, \|}^{2}} \sin ^{2} \varphi\left(1-s^{2}\right) \\
& \left.\times\left[3 s^{2}-\sin ^{2} \varphi\left(1-s^{2}\right)\right]\right]
\end{aligned}
$$

for the first transformation, and

$$
\begin{aligned}
\left(\delta \mathbf{r}_{2}-\delta \mathbf{r}_{1}\right) \cdot \mathbf{r}_{12}= & -\frac{\delta R}{R} r^{2}\left(\frac{R}{r_{1, \|}}\right)^{2}\left[s^{2}-\sin ^{2} \varphi\left(1-s^{2}\right)\right. \\
& -\frac{s r}{r_{1, \|}}\left[s^{2}-3 \sin ^{2} \varphi\left(1-s^{2}\right)\right] \\
& +\frac{r^{2}}{r_{1, \|}^{2}}\left[s^{4}+6 \sin ^{2} \varphi\left(s^{2}-s^{4}\right)\right. \\
& \left.\left.+\sin ^{4} \varphi\left(1-s^{2}\right)^{2}\right]\right]
\end{aligned}
$$

for the second transformation.

Turning first to the spherical droplet, we substitute Eq. (A1), together with Eqs. (A8) and (A9), into Eq. (A6). We then expand the factor of $1 / r_{1}$ in powers of $1 / R$, using the definition of $r_{1} \equiv z_{1}+R$. This leaves us with an expansion in 
powers of $1 / R$, given in the main text as Eq. (2.8). A similar, though slightly more complicated, expansion is used to obtain Eq. (2.9) for the cylindrical interface.

${ }^{1}$ For reviews see Micelles, Membranes, Microemulsions, and Monolayers, edited by W. M. Gelbart, A. Ben-Shaul, and D. Roux (Springer, New York, 1994); Statistical Mechanics of Membranes and Surfaces, edited by D. Nelson, T. Piran, and S. Weinberg (World Scientific, Singapore, 1988); M. Wortis, U. Seifert, K. Berndl, B. Fourcade, M. Rao, and R. Zia, in Dynamical Phenomena at Interfaces, Surfaces and Membranes, edited by D. Beysens, N. Boccara, and G. Forgacs (Nova Science, New York, 1993).

${ }^{2}$ W. Helfrich, Z. Naturforsch. C 28, 693 (1973).

${ }^{3}$ For a review see G. Gompper and M. Schick, Self-assembling Amphiphilic System, Phase Transitions and Critical Phenomena 16, edited by C. Domb and J. Lebowitz (Academic, London, 1994).

${ }^{4}$ E. M. Blokhuis and D. Bedeaux, Physica A 184, 42 (1992); J. Chem. Phys. 95, 6986 (1991); Mol. Phys. 80, 705 (1993); Heterog. Chem. Rev. 1, 55 (1994).

${ }^{5}$ F. Clement and J.-F. Joanny, J. Phys. II 7, 973 (1997).

${ }^{6}$ J. S. Rowlinson, J. Phys.: Condens. Matter 6, 1 (1994).

${ }^{7}$ R. C. Tolman, J. Chem. Phys. 17, 333 (1949).

${ }^{8}$ J. S. Rowlinson and B. Widom, Molecular Theory of Capillarity (Clarendon, Oxford, 1984).

${ }^{9}$ J. W. Gibbs, Collected Works, 2 Vols. (Dover, New York, 1961).

${ }^{10}$ A. Trokhymchuk and J. Alejandre, J. Chem. Phys. 111, 8510 (1999).

${ }^{11}$ C. D. Holcomb, P. Clancy, S. M. Thompson, and J. A. Zollweg, Fluid Phase Equilib. 75, 185 (1992); C. D. Holcomb, P. Clancy, and J. A. Zollweg, Mol. Phys. 78, 437 (1993).

${ }^{12}$ A. E. van Giessen, E. M. Blokhuis, and D. J. Bukman, J. Chem. Phys.
108, 1148 (1998); L. Granasy, ibid. 109, 9660 (1998); J. Barrett, ibid. 111, 5938 (1999); K. Koga, X. C. Zeng, and A. K. Schekin, ibid. 109, 4063 (1998); T. V. Bykov and A. K. Schekin, Colloid J. 61, 144 (1999); Inorg. Mater. (Transl. of Neorg. Mater.) 35, 641 (1999); T. V. Bykov and X. C. Zeng, J. Chem. Phys. 111, 3705 (1999); 111, 10602 (1999); V. G. Baidakov and G. Sh. Boltachev, Phys. Rev. E 59, 469 (1999).

${ }^{13}$ J. G. Kirkwood and F. P. Buff, J. Chem. Phys. 17, 338 (1949).

${ }^{14}$ M. J. Haye and C. Bruin, J. Chem. Phys. 100, 556 (1994).

${ }^{15}$ M. J. P. Nijmeijer, A. F. Bakker, C. Bruin, and J. H. Sikkenk, J. Chem. Phys. 89, 3789 (1988); E. M. Blokhuis, D. Bedeaux, C. D. Holcomb, and J. A. Zollweg, Mol. Phys. 85, 665 (1995).

${ }^{16}$ M. Mareschal, M. Baus, and R. Lovett, J. Chem. Phys. 106, 645 (1997).

${ }^{17}$ H. El Bardouni, M. Mareschal, R. Lovett, and M. Baus, J. Chem. Phys. 113, 9804 (2000).

${ }^{18}$ J. S. Rowlinson, J. Phys. A 17, L357 (1984).

${ }^{19}$ M. P. A. Fisher and M. Wortis, Phys. Rev. B 29, 6252 (1984).

${ }^{20}$ E. M. Blokhuis, J. Groenewold, and D. Bedeaux, Mol. Phys. 96, 397 (1999)

${ }^{21}$ H. Kellay, B. P. Binks, and J. Meunier, Phys. Rev. Lett. 70, 1485 (1993).

${ }^{22}$ M. Borkovec and H.-F. Eicke, Chem. Phys. Lett. 157, 457 (1989); J. S. Huang, S. T. Milner, B. Farargo, and D. Richter, Phys. Rev. Lett. 59, 2600 (1987); B. P. Binks, J. Meunier, O. Abillon, and D. Langevin, Langmuir 5, 415 (1989).

${ }^{23}$ J. W. Schmidt, Phys. Rev. A 38, 567 (1988); E. M. Blokhuis and D. Bedeaux, Physica A 164, 515 (1990).

${ }^{24}$ J. Daillant and M. Alba, Rep. Prog. Phys. 63, 1725 (2000); C. Fradin, A. Braslau, D. Luzet, D. Smilgies, M. Alba, N. Boudet, K. Mecke, and J. Daillant, Nature (London) 403, 871 (2000).

${ }^{25}$ K. R. Mecke and S. Dietrich, Phys. Rev. E 59, 6766 (1999). 